\title{
2-氭基-3-取代丙烯酸(2-乙氧基乙基)酯的合成及除草活性
}

\author{
钟世华王春凤宋青霞范明亮刘兵玉危冬梅 \\ 刘建兵* \\ ( ${ }^{a}$ 石化新材料与资源精细利用国家地方联合工程实验室 化学生物学及中药分析省部共建教育部重点实验室 \\ 湖南师范大学 长沙 410081)
}

\begin{abstract}
摘要 设计并合成了 11 个 2-氭基-3-取代苯胺基-3-甲硫基丙烯酸(2-乙氧基乙基)酯(2)和 11 个 2-氰基-3-取代苯基腿基丙 烯酸(2-乙氧基乙基)酯(5)两类光合作用光系统 II (PSII)电子传递抑制剂，目标化合物的结构均经 ${ }^{1} \mathrm{H} N \mathrm{NMR},{ }^{13} \mathrm{C} \mathrm{NMR}$ 和 质谱分析确证，生物活性测试结果表明：部分化合物 2 对双子叶杂草表现出良好的选择性和除草活性，化合物 $\mathbf{5}$ 苯环上 间位有取代基时有一定的除草活性.
\end{abstract}

关键词＼cjkstart氭基丙烯酸酯; 苯基脲; PSII 抑制剂; 除草活性

\section{Synthesis and Herbicidal Activities of 2-Ethoxyethyl 2-Cyano-3-(substituted)acrylates}

\author{
Zhong, Shihua $\quad$ Wang, Chunfeng Song, Qingxia Fan, Mingliang Liu, Bingyu \\ Wei, Dongmei Liu, Jianbing* \\ (National \& Local Joint Engineering Laboratory for New Petro-chemical Materials and Fine Utilization of Resources, Key \\ Laboratory of Chemical Biology and Traditional Chinese Medicine Research, Ministry of Education, \\ Hunan Normal University, Changsha 410081)
}

\begin{abstract}
Eleven new 2-ethoxyethyl 2-cyano-3-(substituted)anilino-3-methylthio acrylates (2) and eleven new 2-ethoxyethyl 2-cyano-3-(substituted)phenylureidoacrylates (5) were synthesized as herbicidal inhibitors of photosystem II (PSII) electron transport, and their structures were confirmed by ${ }^{1} \mathrm{H}$ NMR, ${ }^{13} \mathrm{C}$ NMR and mass spectrum analysis. Biological activity tests showed that some of compounds $\mathbf{2}$ have good selectivity and activity against dicotyledonous weeds. Compounds $\mathbf{5}$ bearing a group at the 3-position in benzene ring exhibite some degree of herbicidal activities.
\end{abstract}

Keywords cyanoacrylate; phenylurea; inhibitor of PSII; herbicidal activity

氧基丙烯酸酯类化合物具有广泛的生物活性，可用 作除草剂、杀虫剂、杀菌剂等 ${ }^{[1 \sim 3]}$, 其除草活性是除草 剂研究的热点之一 ${ }^{[4 \sim 8]}$. 该类化合物除草机理为竞争性 抑制光合作用电子传递链上膜蛋白复合物光系统 II (PSII)中的 D1 蛋白, 破坏电子传递, 从而抑制杂草的光 合作用, 达到除草效果 ${ }^{[9]}$. 苯基艮类化合物同样具有除 草活性, 其除草机理与氧基丙烯酸酯类化合物相似. 为 了寻找高除草活性的氧基丙烯酸酯类化合物, 我们在不 改变氰基丙烯酸酯母体结构(图 1)的前提下, 利用生物 电子等排原理，用芳 胺基替代苄基，设计并合成了 2氧基-3-取代苯胺基-3-甲硫基丙烯酸(2-乙氧基乙基)酯<smiles>[R]C(=O)/C(C#N)=C(\[R])NCc1ccc([R])cc1</smiles>

$\mathrm{R}^{1}=\mathrm{F}, \mathrm{Cl}, \mathrm{CH}_{3}$, etc.;

$\mathrm{R}^{2}=\mathrm{SCH}_{3}, \mathrm{C}_{2} \mathrm{H}_{5}, \mathrm{H}$, etc.;

$\mathrm{R}^{3}=\mathrm{C}_{2} \mathrm{H}_{5}, \mathrm{C}_{2} \mathrm{H}_{4} \mathrm{OC}_{2} \mathrm{H}_{5}$, etc.

图 1 氧基丙烯酸酯类化合物的结构式

Figure 1 The structure of cyanoacrylate

(2) (Scheme 1); 采用活性亚结构拼接法, 将苯基脲结构 单元与氧基丙烯酸酯对接设计合成了 2-氧基-3-取代苯

*E-mail: liu_jianbing65@yahoo.com.cn

Received April 12, 2014;; revised June 5, 2014; published online July 1, 2014.

Project supported by the National Natural Science Foundation of China (No. 20602010).

国家自然科学基金(No. 20602010)资助项目. 
基嘱基丙烯酸(2-乙氧基乙基)酯(5). 目标化合物的合成 方法如 Scheme 1 所示. 生物活性测试结果表明: 化合物 $2 \mathbf{c}$ 和 $2 \mathbf{i}$ 对苟麻、刺苋和僽显示 $80 \%$ 的除草活性, 而对马 唐、稗和狗尾不具除草活性; 化合物 $\mathbf{5 c}, \mathbf{5 f}$ 和 $\mathbf{5 k}$ 有一定 的除草活性.<smiles>CCOC(=O)C(C)=C(C)C(=O)OCC</smiles>

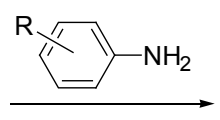<smiles>[R]c1ccc(NC(C)=C(C#N)C(=O)OCOC)cc1</smiles>
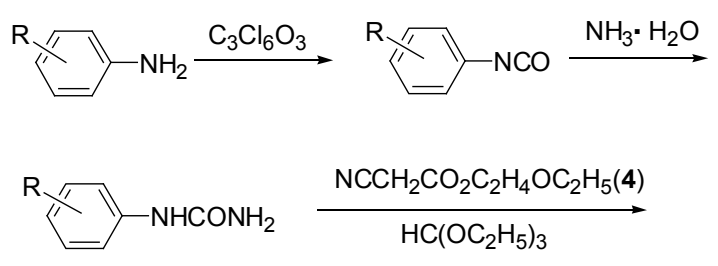

3

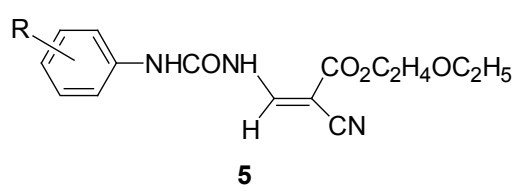

$\mathrm{R}=\mathrm{H}(\mathbf{a}), 2-\mathrm{CH}_{3}(\mathbf{b}), 3-\mathrm{CH}_{3}(\mathbf{c}), 4-\mathrm{CH}_{3}(\mathbf{d}), 2-\mathrm{Cl}(\mathbf{e}), 3-\mathrm{Cl}(\mathbf{f}), 4-\mathrm{Cl}(\mathbf{g})$, 2-F (h), 4-F (i), 2,4-F $(\mathrm{j}), 4-\mathrm{F}-3-\mathrm{Cl}(\mathbf{k})$

\section{Scheme 1}

\section{1 结果与讨论}

\section{1 目标化合物的合成}

化合物 2 由中间体 1 与取代苯胺加热回流合成, $\mathrm{TLC}$ 检测反应进度时, 中间体 $\mathbf{1}$ 与化合物 $\mathbf{2}$ 的 $R_{\mathrm{f}}$ 值很相 近, 不易观察. 加料时可适当使取代苯胺过量, 使其与 中间体 1 的摩尔比为 $1.3: 1$ 或 $1.4: 1$, 使中间体 1 尽可 能反应完全. 在化合物 $\mathbf{5}$ 的合成过程中, 产物可能会有 $E$ 式和 $Z$ 式两种构型, 用乙醇重结晶恰好可以得到以 $\mathrm{Z}$ 式构型为主的产物.

化合物 5 在 $\left(\mathrm{CD}_{3}\right)_{2} \mathrm{SO}$ 中溶解性很好, 但易变质, 需 配好溶液后尽快进行核磁分析, 在 $\left(\mathrm{CD}_{3}\right)_{2} \mathrm{CO}$ 中溶解性 一般, 但不易变质. 将化合物 5 溶于 $\left(\mathrm{CD}_{3}\right)_{2} \mathrm{SO}$ 中, 放置 一段时间再进行 ${ }^{1} \mathrm{H}$ NMR 分析, 核磁图中可以发现在脲 基中的两个氢原子和双键碳上的氢原子对应峰的旁边 会明显出现峰形相似的小杂质峰, 我们推测在 $\left(\mathrm{CD}_{3}\right)_{2} \mathrm{SO}$ 中化合物 $\mathbf{5}$ 存在烯胺-亚胺互变, 从而导致化合物 $\mathbf{5}$ 从单 一的 $Z$ 式结构变为 $E$ 式 $Z$ 式共存. $E$ 式 $Z$ 式互变过程如 图 2 所示.<smiles>CC=C(C)C=[NH+]C(=O)C(C)CC</smiles><smiles>CC(=O)N/C=C(\C)C(C)(C)C</smiles>

图 2 目标化合物的顺反异构

Figure 2 Cis-trans isomerization of title compounds

\section{2 化合物 $5 a$ 的晶体结构分析}

为验证目标化合物 $\mathbf{5}$ 用乙醇重结晶后得到的产物为 $Z$ 式构型，我们选取化合物 $\mathbf{5 a}$ 进行了 $X$ 射线单晶衍射分 析.

将经过两次乙醇重结晶提纯后的目标化合物 $\mathbf{5 a}$ 溶 于体积比为 $1: 2$ 的乙醇和丙酮混合溶液中, 室温静置 使其缓慢挥发, 数天后形成无色片状晶体, 选取 0.48 $\mathrm{mm} \times 0.44 \mathrm{~mm} \times 0.09 \mathrm{~mm}$ 的单晶进行 $X$ 射线衍射分析 (CCDC 号为 1001714). 结果表明, 化合物 5a 为 $Z$ 式构 型, 该化合物分子式为: $\mathrm{C}_{15} \mathrm{H}_{17} \mathrm{~N}_{3} \mathrm{O}_{4}$, 该晶体属于单斜 晶系, 空间群为 $C 2 / c$, 晶胞参数为 $a=2.5102(7) \mathrm{nm}, b=$ 1.2013(3) nm, $c=1.0436(3) \mathrm{nm}, \alpha=90.00^{\circ}, \beta=$ $96.248(4)^{\circ}, \gamma=90.00^{\circ}, V=3.1284(16) \mathrm{nm}^{3} . Z=8, D_{\mathrm{c}}=$ $1.288 \mathrm{~g} / \mathrm{cm}^{3}, \mu=0.095 \mathrm{~mm}^{-1}, F(000)=1280, R=0.043$, $w R=0.116$. 图 3 为化合物 $\mathbf{5 a}$ 的分子结构图.

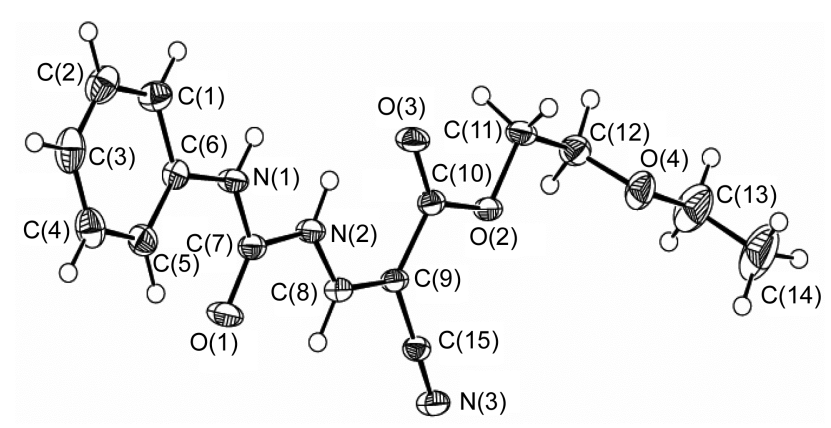

图 3 化合物 $5 \mathrm{a}$ 的分子结构图

Figure 3 Molecular structure of compound 5a

\section{3 生物活性测试及构效关系}

我们对化合物 $\mathbf{2 a} \sim \mathbf{2 k}$, 化合物 $\mathbf{5 a} \sim \mathbf{5 k}$ 进行了除草 活性普篮, 测试杂草有双子叶杂草: 苟麻(Abutilon theophrasti)、刺苋 (Amaranthus spinosus L)、菉 (Chenopodium album) 和单子叶杂草：马唐(Digitaria sanguinalis)、稗(Echinochloa crus-galli)、狗尾(Setaria viridis).

在塑料盆钵中定量装土压平, 置于不锈钢盆中, 将 杂草种子按单、双子叶植物分钵播种, 覆 $1 \mathrm{~cm}$ 厚细土, 
从塑料盆钵底部加水至上层土壤浸润, 置于温室培养. 将提纯后的化合物溶于适量 $\mathrm{DMF}$, 加入少量乳化剂 Tween20, 再加清水稀释到适当浓度, 待单子叶植物长 至 1 叶 1 心期、双子叶植物长至 2 片真叶期进行苗后茎 叶处理, 处理剂量为 $2.25 \mathrm{~kg} / \mathrm{ha} .20 \mathrm{~d}$ 后观察靶标发芽及 生长情况, 记录试验结果. 试验设清水对照组和溶剂对 照组. 测试结果参见表 1(未列出的化合物不显示除草活 性).

表 1 目标化合物的除草活性

Table 1 Herbicidal activities of title compounds

\begin{tabular}{ccccccc}
\hline Compd. & 苟麻 & 刺苋 & 藜 & 马唐 & 稗 & 狗尾 \\
\hline $\mathbf{2 c}$ & 80 & 80 & 80 & 0 & 0 & 0 \\
$\mathbf{2 d}$ & 50 & 50 & 50 & 0 & 0 & 0 \\
$\mathbf{2 g}$ & 0 & 0 & 0 & 30 & 0 & 0 \\
$\mathbf{2 i}$ & 85 & 85 & 85 & 0 & 0 & 0 \\
$\mathbf{5 c}$ & 0 & 0 & 0 & 40 & 0 & 50 \\
$\mathbf{5 f}$ & 0 & 0 & 70 & 0 & 0 & 0 \\
$\mathbf{5 k}$ & 0 & 0 & 70 & 0 & 0 & 0 \\
\hline
\end{tabular}

由表 1 可知, 化合物 $\mathbf{2 a} \sim \mathbf{2 k}$ 中大多数无除草活性, 进一步证实了烯氨基和苯环之间加入亚甲基的必要性, 亚甲基的加入可以使苯环更好的与 D1 蛋白上 Phe 211, Phe 255, Phe 265 和 Phe 274 产生范德华力和 $\pi$-堆积相互 作用. 但化合物 2c, 2d 和 2i 对双子叶杂草表现出较高的 除草活性和很好的选择性, 可以发现此类化合物中苯环 4-位有适当取代基将有助于除草活性的提高.

对化合物 $5 \mathrm{a} \sim 5 \mathrm{k}$, 苯基脲的引入并没使其除草活 性得到预期的提高, 在苯环 3-位有取代基时化合物才表 现出除草活性，而且当 $\mathrm{R}$ 基团为甲基时，化合物对单子 叶杂草有一定的抑制作用, 当 $\mathrm{R}$ 基团为卤素时, 化合物 对双子叶杂草有较好的除草效果. 我们推测苯基䐂基中 的氧原子而非 $\alpha$-位羰基上的氧原子与 Ser 264 形成氢键, 主要是苯基脲活性基团在显示除草效果, 由于苯基脲类 化合物的除草活性一般比氰基丙烯酸酯类化合物效果 差 ${ }^{[10]}$, 所以苯基腿活性基团的引入使㲵基丙烯酸酯活 性基团所起的除草作用减弱，导致化合物除草效果不甚 理想.

\section{2 实验部分}

\section{1 试剂与仪器}

Bruker 500 型核磁共振仪, 内标为 TMS; SHIMADZU GCMS-QP 2010 质谱测量仪; 天大科技有限公司 YRT-3 型熔点仪, 温度计未经校正; 柱层析硅胶为青岛 海洋化工厂出品; 实验用试剂与原料均为分析纯或化学 纯(其中石油醚的沸程为 $60 \sim 90{ }^{\circ} \mathrm{C}$ ). 中间体 2-氧基3,3-二甲硫基丙烯酸(2-乙氧基乙基)酯(1), 㲵乙酸乙氧 乙酯(4)均根据参考文献[11]制备.

\section{2 目标化合物的合成}

2.2.1 2-氰基-3-取代苯胺基-3-甲硫基丙烯酸 (2-乙氧 基乙基)酯( $(2 \mathrm{a} \sim 2 \mathrm{k})$ 的合成

于装有电动摚拌的 $25 \mathrm{~mL}$ 三口反应瓶中, 依次加入 $0.52 \mathrm{~g}(2.0 \mathrm{mmol}) 2$-氯基-3,3-二甲硫基丙烯酸(2-乙氧基 乙基)酯(1)、2.4 $\mathrm{mmol}$ 取代苯胺和 $10 \mathrm{~mL}$ 乙醇, 加热回 流至原料点消失后停止反应(TLC 检测), 浓缩, 残余物 用乙醇重结晶得目标产物 ${ }^{[1]}$. 目标化合物的结构经 ${ }^{1} \mathrm{H}$ $\mathrm{NMR},{ }^{13} \mathrm{C}$ NMR 和质谱分析确证.

2-氧基-3-苯胺基-3-甲硫基丙烯酸(2-乙氧基乙基)酯 (2a): 白色固体，收率 $85 \%$. m.p. $67.5 \sim 68.6{ }^{\circ} \mathrm{C} ;{ }^{1} \mathrm{H}$ NMR $\left(500 \mathrm{MHz}, \mathrm{CDCl}_{3}\right) \delta: 1.14\left(\mathrm{t}, J=7.0 \mathrm{~Hz}, 3 \mathrm{H}, \mathrm{CH}_{3}\right)$, $2.16\left(\mathrm{~s}, 3 \mathrm{H}, \mathrm{SCH}_{3}\right), 3.50$ (q, $\left.J=7.0 \mathrm{~Hz}, 2 \mathrm{H}, \mathrm{OCH}_{2}\right), 3.64$ (t, $\left.J=5.0 \mathrm{~Hz}, 2 \mathrm{H}, \mathrm{CH}_{2} \mathrm{O}\right), 4.27$ (t, $J=5.0 \mathrm{~Hz}, 2 \mathrm{H}$, $\left.\mathrm{CO}_{2} \mathrm{CH}_{2}\right), 7.21 \sim 7.35\left(\mathrm{~m}, 5 \mathrm{H}, \mathrm{C}_{6} \mathrm{H}_{5}\right), 11.37(\mathrm{~s}, 1 \mathrm{H}, \mathrm{NH})$; ${ }^{13} \mathrm{C}$ NMR $\left(125 \mathrm{MHz}, \mathrm{CDCl}_{3}\right) \delta: 15.2,17.3,64.4,66.8$, 68.0, 77.2, 117.4, 124.9, 127.3, 129.5, 137.6, 167.7, 170.4; MS (EI, $70 \mathrm{eV}$ ) $m / z(\%): 306\left(\mathrm{M}^{+}, 22\right), 259$ (17), $216(30)$, 187 (13), 169 (100), 144 (9), 77 (23), 45 (34), 29 (10). Anal. calcd for $\mathrm{C}_{15} \mathrm{H}_{18} \mathrm{~N}_{2} \mathrm{O}_{3} \mathrm{~S}$ : C 58.80, H 5.92, N 9.14; found C 58.62, H 5.78, N 9.05.

2-氧基-3-(2-甲基)苯胺基-3-甲硫基丙烯酸 (2-乙氧 基乙基)酯(2b): 白色固体，收率 73\%. m.p. 76.0 78.3 ${ }^{\circ} \mathrm{C} ;{ }^{1} \mathrm{H}$ NMR $\left(500 \mathrm{MHz}, \mathrm{CDCl}_{3}\right) \delta: 1.15(\mathrm{t}, J=7.0 \mathrm{~Hz}$, $\left.3 \mathrm{H}, \mathrm{CH}_{3}\right), 2.18\left(\mathrm{~s}, 3 \mathrm{H}, \mathrm{SCH}_{3}\right), 2.21\left(\mathrm{~s}, 3 \mathrm{H}, \mathrm{CH}_{3} \mathrm{C}_{6} \mathrm{H}_{4}\right), 3.52$ (q, $J=7.0 \mathrm{~Hz}, 2 \mathrm{H}, \mathrm{OCH}_{2}$ ), 3.65 (t, $J=5.0 \mathrm{~Hz}, 2 \mathrm{H}, \mathrm{CH}_{2} \mathrm{O}$ ), $4.27\left(\mathrm{t}, J=4.5 \mathrm{~Hz}, 2 \mathrm{H}, \mathrm{CO}_{2} \mathrm{CH}_{2}\right), 7.16 \sim 7.20(\mathrm{~m}, 4 \mathrm{H}$, $\left.\mathrm{C}_{6} \mathrm{H}_{4}\right), 11.23(\mathrm{~s}, 1 \mathrm{H}, \mathrm{NH}) ;{ }^{13} \mathrm{C}$ NMR $\left(125 \mathrm{MHz}, \mathrm{CDCl}_{3}\right) \delta$ : 15.4, 17.4, 18.2, 64.5, 67.1, 68.3, 77.0, 117.7, 126.6, 127.1, 128.2, 131.2, 133.9, 136.7, 168.2, 171.9; MS (EI $70 \mathrm{eV}$ ) m/z (\%): 320 (M $\left.\mathrm{M}^{+}, 26\right), 273$ (19), 230 (38), 215 (8), 201 (12), 183 (100), 155 (35), 130 (15), 116 (13), 91(22), 73 (18), 45 (46), 29 (12). Anal. calcd for $\mathrm{C}_{16} \mathrm{H}_{20} \mathrm{~N}_{2} \mathrm{O}_{3} \mathrm{~S}$ : C 59.98, H 6.29, N 8.74; found C 59.75, H 6.18, N 8.59.

2-氧基-3-(3-甲基)苯胺基-3-甲硫基丙烯酸 (2-乙氧 基乙基)酯(2c): 白色固体，收率 71\%. m.p. 54.5 55.6 ${ }^{\circ} \mathrm{C} ;{ }^{1} \mathrm{H}$ NMR $\left(500 \mathrm{MHz}, \mathrm{CDCl}_{3}\right) \delta: 1.14(\mathrm{t}, J=7.0 \mathrm{~Hz}, 3 \mathrm{H}$, $\mathrm{CH}_{3}$ ), 2.18 (s, $3 \mathrm{H}, \mathrm{SCH}_{3}$ ), 2.30 (s, $\left.3 \mathrm{H}, \mathrm{CH}_{3} \mathrm{C}_{6} \mathrm{H}_{4}\right), 3.51$ (q, $\left.J=7.0 \mathrm{~Hz}, 2 \mathrm{H}, \mathrm{OCH}_{2}\right), 3.64\left(\mathrm{t}, J=5.0 \mathrm{~Hz}, 2 \mathrm{H}, \mathrm{CH}_{2} \mathrm{O}\right.$ ), $4.27\left(\mathrm{t}, J=5.0 \mathrm{~Hz}, 2 \mathrm{H}, \mathrm{CO}_{2} \mathrm{CH}_{2}\right), 7.01 \sim 7.22(\mathrm{~m}, 4 \mathrm{H}$, $\left.\mathrm{C}_{6} \mathrm{H}_{4}\right), 11.34$ (s, $\left.1 \mathrm{H}, \mathrm{NH}\right) ;{ }^{13} \mathrm{C}$ NMR $\left(125 \mathrm{MHz}, \mathrm{CDCl}_{3}\right) \delta$ : 15.0, 17.2, 21.1, 64.2, 66.7, 67.9, 76.9, 117.3, 121.7, 125.2, $127.9,129.1,137.3,139.4,167.5,170.3$; MS (EI, $70 \mathrm{eV}$ ) m/z (\%): $320\left(\mathrm{M}^{+}, 26\right), 273$ (16), 230 (27), 216 (6), 201 (12), 183 (100), 155 (11), 91 (31), 73 (16), 45 (44), 29 
(10). Anal. calcd for $\mathrm{C}_{16} \mathrm{H}_{20} \mathrm{~N}_{2} \mathrm{O}_{3} \mathrm{~S}$ : C 59.98, H 6.29, N 8.74; found C 59.81, H 6.16, N 8.64.

2-氧基-3-(4-甲基)苯胺基-3-甲硫基丙烯酸(2-乙氧 基乙基)酯(2d)：白色固体，收率 77\%. m.p. 72.3 74.6 ${ }^{\circ} \mathrm{C} ;{ }^{1} \mathrm{H}$ NMR $\left(500 \mathrm{MHz}, \mathrm{CDCl}_{3}\right) \delta: 1.15(\mathrm{t}, J=7.0 \mathrm{~Hz}, 3 \mathrm{H}$, $\mathrm{CH}_{3}$ ), 2.19 (s, 3H, $\mathrm{SCH}_{3}$ ), 2.30 (s, 3H, $\left.\mathrm{CH}_{3} \mathrm{C}_{6} \mathrm{H}_{4}\right), 3.52$ (q, $\left.J=7.0 \mathrm{~Hz}, 2 \mathrm{H}, \mathrm{OCH}_{2}\right), 3.65$ (t, $J=5.0 \mathrm{~Hz}, 2 \mathrm{H}, \mathrm{CH}_{2} \mathrm{O}$ ), $4.27\left(\mathrm{t}, J=5.0 \mathrm{~Hz}, 2 \mathrm{H}, \mathrm{CO}_{2} \mathrm{CH}_{2}\right), 7.08 \sim 7.20(\mathrm{~m}, 4 \mathrm{H}$, $\left.\mathrm{C}_{6} \mathrm{H}_{4}\right), 11.34$ (s, $\left.1 \mathrm{H}, \mathrm{NH}\right) ;{ }^{13} \mathrm{C}$ NMR $\left(125 \mathrm{MHz}, \mathrm{CDCl}_{3}\right) \delta$ : 15.1, 17.3, 21.0, 64.3, 66.8, 68.0, 76.9, 117.5, 124.9, 130.0, 135.0, 137.4, 167.8, 170.6; MS (EI, $70 \mathrm{eV}) \mathrm{m} / \mathrm{z}(\%): 320$ $\left(\mathrm{M}^{+}, 25\right), 273$ (10), 230 (40), 216 (5), 200 (11), 183 (100), 155 (13), 130 (5), 91 (16), 73 (12), 45 (30), 29 (8). Anal. calcd for $\mathrm{C}_{16} \mathrm{H}_{20} \mathrm{~N}_{2} \mathrm{O}_{3} \mathrm{~S}$ : C 59.98, H 6.29, N 8.74; found C 59.88, H 6.20, N 8.59.

2-氧基-3-(2-氯)苯胺基-3-甲硫基丙烯酸 (2-乙氧基 乙基)酯(2e): 白色固体; 收率 70\%. m.p. 81.0 82.0 ${ }^{\circ} \mathrm{C}$; ${ }^{1} \mathrm{H}$ NMR $\left(500 \mathrm{MHz}, \mathrm{CDCl}_{3}\right) \delta: 1.14(\mathrm{t}, J=7.0 \mathrm{~Hz}, 3 \mathrm{H}$, $\left.\mathrm{CH}_{3}\right), 2.18$ (s, $\left.3 \mathrm{H}, \mathrm{SCH}_{3}\right), 3.51$ (q, J=7.0 Hz, $2 \mathrm{H}, \mathrm{OCH}_{2}$ ), 3.65 (t, $\left.J=5.0 \mathrm{~Hz}, 2 \mathrm{H}, \mathrm{CH}_{2} \mathrm{O}\right), 4.29$ (t, $J=5.0 \mathrm{~Hz}, 2 \mathrm{H}$, $\left.\mathrm{CO}_{2} \mathrm{CH}_{2}\right), 7.18 \sim 7.42\left(\mathrm{~m}, 4 \mathrm{H}, \mathrm{C}_{6} \mathrm{H}_{4}\right), 11.22(\mathrm{~s}, 1 \mathrm{H}, \mathrm{NH})$; ${ }^{13} \mathrm{C}$ NMR (125 MHz, $\left.\mathrm{CDCl}_{3}\right) \delta: 15.1,17.1,64.5,66.7$, 67.9, 78.7, 117.0, 127.0, 127.5, 128.4,129.9, 130.1, 135.1, 167.3, 170.6; MS (EI, $70 \mathrm{eV}) \mathrm{m} / \mathrm{z}(\%): 340\left(\mathrm{M}^{+}, 23\right), 305$ (23), 293 (22), 250 (41), 215 (18), 203 (100), 178 (16), 111 (17), 73 (39), 45 (78), 29 (21). Anal. calcd for $\mathrm{C}_{15} \mathrm{H}_{17} \mathrm{Cl}$ $\mathrm{N}_{2} \mathrm{O}_{3} \mathrm{~S}: \mathrm{C} 52.86, \mathrm{H} 5.03, \mathrm{~N} 8.22$; found C 52.74, H 4.95, N 8.06 .

2-氰基-3-(3-氯)苯胺基-3-甲硫基丙烯酸(2-乙氧基 乙基)酯(2f): 白色固体, 收率 61\%. m.p. 82.0 $82.7{ }^{\circ} \mathrm{C}$; ${ }^{1} \mathrm{H}$ NMR $\left(500 \mathrm{MHz}, \mathrm{CDCl}_{3}\right) \delta: 1.15(\mathrm{t}, J=7.0 \mathrm{~Hz}, 3 \mathrm{H}$, $\left.\mathrm{CH}_{3}\right), 2.23$ (s, $\left.3 \mathrm{H}, \mathrm{SCH}_{3}\right), 3.51$ (q, $J=7.0 \mathrm{~Hz}, 2 \mathrm{H}, \mathrm{OCH}_{2}$ ), $3.65\left(\mathrm{t}, J=5.0 \mathrm{~Hz}, 2 \mathrm{H}, \mathrm{CH}_{2} \mathrm{O}\right), 4.28(\mathrm{t}, J=5.0 \mathrm{~Hz}, 2 \mathrm{H}$, $\mathrm{CO}_{2} \mathrm{CH}_{2}$ ), $7.12 \sim 7.29\left(\mathrm{~m}, 4 \mathrm{H}, \mathrm{C}_{6} \mathrm{H}_{4}\right), 11.33(\mathrm{~s}, 1 \mathrm{H}, \mathrm{NH})$; ${ }^{13} \mathrm{C}$ NMR $\left(125 \mathrm{MHz}, \mathrm{CDCl}_{3}\right) \delta: 15.1,17.3,64.5,66.8$, 67.9, 78.6, 116.9, 122.9, 124.9, 127.2, 130.4, 135.0, 138.8, 167.4, 170.1; MS (EI, $70 \mathrm{eV}) \mathrm{m} / \mathrm{z}(\%): 340\left(\mathrm{M}^{+}, 24\right), 293$ (25), 250 (55), 203 (100), 178 (16), 111 (25), 73 (45), 45 (76), 29 (20). Anal. calcd for $\mathrm{C}_{15} \mathrm{H}_{17} \mathrm{ClN}_{2} \mathrm{O}_{3} \mathrm{~S}$ : C 52.86, H 5.03, N 10.40; found C 52.79, H 4.88, N 8.08.

2-氧基-3-(4-氯)苯胺基-3-甲硫基丙烯酸 (2-乙氧基 乙基)酯(2g): 白色固体, 收率 82\%. m.p. 67.1 67.9 ${ }^{\circ} \mathrm{C}$; ${ }^{1} \mathrm{H}$ NMR $\left(500 \mathrm{MHz}, \mathrm{CDCl}_{3}\right) \delta: 1.14(\mathrm{t}, J=7.0 \mathrm{~Hz}, 3 \mathrm{H}$, $\left.\mathrm{CH}_{3}\right), 2.21\left(\mathrm{~s}, 3 \mathrm{H}, \mathrm{SCH}_{3}\right), 3.51$ (q, J=7.0 Hz, $2 \mathrm{H}, \mathrm{OCH}_{2}$ ), $3.64\left(\mathrm{t}, \mathrm{J}=5.0 \mathrm{~Hz}, 2 \mathrm{H}, \mathrm{CH}_{2} \mathrm{O}\right), 4.27(\mathrm{t}, J=5.0 \mathrm{~Hz}, 2 \mathrm{H}$,
$\left.\mathrm{CO}_{2} \mathrm{CH}_{2}\right), 7.16 \sim 7.31\left(\mathrm{~m}, 4 \mathrm{H}, \mathrm{C}_{6} \mathrm{H}_{4}\right), 11.32(\mathrm{~s}, 1 \mathrm{H}, \mathrm{NH})$; ${ }^{13} \mathrm{C}$ NMR $\left(125 \mathrm{MHz}, \mathrm{CDCl}_{3}\right) \delta: 15.2,17.4,64.5,66.9$, 68.0, 78.2, 117.1, 126.2, 129.6, 132.9, 136.2, 167.6, 170.2; MS (EI, $70 \mathrm{eV}) \mathrm{m} / z$ (\%): 340 (M+ , 32), 293 (14), 250 (84), 203 (100), 175 (14), 111 (19), 73 (33), 45 (70), 32 (61), 29 (24). Anal. calcd for $\mathrm{C}_{15} \mathrm{H}_{17} \mathrm{ClN}_{2} \mathrm{O}_{3} \mathrm{~S}$ : C 52.86, H 5.03, N 10.40; found C 52.74, H 4.95, N 8.13.

2-氭基-3-(2-氟)苯胺基-3-甲硫基丙烯酸 (2-乙氧基 乙基)酯(2h): 白色固体，收率 70\%. m.p. $64.5 \sim 65.0{ }^{\circ} \mathrm{C}$; ${ }^{1} \mathrm{H}$ NMR $\left(500 \mathrm{MHz}, \mathrm{CDCl}_{3}\right) \delta: 1.15(\mathrm{t}, J=7.0 \mathrm{~Hz}, 3 \mathrm{H}$, $\left.\mathrm{CH}_{3}\right), 2.30$ (s, $3 \mathrm{H}, \mathrm{SCH}_{3}$ ), 3.52 (q, $J=7.0 \mathrm{~Hz}, 2 \mathrm{H}, \mathrm{OCH}_{2}$ ), $3.66\left(\mathrm{t}, J=5.0 \mathrm{~Hz}, 2 \mathrm{H}, \mathrm{CH}_{2} \mathrm{O}\right), 4.29(\mathrm{t}, J=5.0 \mathrm{~Hz}, 2 \mathrm{H}$, $\left.\mathrm{CO}_{2} \mathrm{CH}_{2}\right), 7.09 \sim 7.34\left(\mathrm{~m}, 4 \mathrm{H}, \mathrm{C}_{6} \mathrm{H}_{4}\right), 11.15(\mathrm{~s}, 1 \mathrm{H}, \mathrm{NH})$; ${ }^{13} \mathrm{C}$ NMR $\left(125 \mathrm{MHz}, \mathrm{CDCl}_{3}\right) \delta: 14.1,16.4,63.5,65.9$, 67.0, 77.7, 115.4 (d, $J=19.9 \mathrm{~Hz}), 116.1,123.6$ (d, $J=3.8$ Hz), $124.7(\mathrm{~d}, J=12.1 \mathrm{~Hz}), 126.3,128.0(\mathrm{~d}, J=7.3 \mathrm{~Hz})$, 154.5 (d, $J=248.3 \mathrm{~Hz}), 166.5$, 170.4; MS (EI, $70 \mathrm{eV}$ ) $\mathrm{m} / \mathrm{z}$ (\%): $324\left(\mathrm{M}^{+}, 20\right), 277$ (11), 234 (61), 205 (9), 187 (100), 159 (20), 110 (5), 95 (20), 75 (19), 45 (51), 32 (63). Anal. calcd for $\mathrm{C}_{15} \mathrm{H}_{17} \mathrm{FN}_{2} \mathrm{O}_{3} \mathrm{~S}$ : C 55.54, H 5.28, N 8.64; found $\mathrm{C}$ 55.37, H 5.10, N 8.50.

2-氧基-3-(4-氟)苯胺基-3-甲硫基丙烯酸 (2-乙氧基 乙基)酯(2i): 白色固体, 收率 79\%. m.p. $67.5 \sim 69.5{ }^{\circ} \mathrm{C}$; ${ }^{1} \mathrm{H}$ NMR $\left(500 \mathrm{MHz}, \mathrm{CDCl}_{3}\right) \delta: 1.14(\mathrm{t}, J=7.0 \mathrm{~Hz}, 3 \mathrm{H}$, $\mathrm{CH}_{3}$ ), 2.21 (s, $3 \mathrm{H}, \mathrm{SCH}_{3}$ ), 3.51 (q, J=7.0 Hz, $2 \mathrm{H}, \mathrm{OCH}_{2}$ ), $3.65\left(\mathrm{t}, J=5.0 \mathrm{~Hz}, 2 \mathrm{H}, \mathrm{CH}_{2} \mathrm{O}\right), 4.27(\mathrm{t}, J=5.0 \mathrm{~Hz}, 2 \mathrm{H}$, $\left.\mathrm{CO}_{2} \mathrm{CH}_{2}\right), 7.02 \sim 7.21\left(\mathrm{~m}, 4 \mathrm{H}, \mathrm{C}_{6} \mathrm{H}_{4}\right), 11.31(\mathrm{~s}, 1 \mathrm{H}, \mathrm{NH})$; ${ }^{13} \mathrm{C}$ NMR (125 MHz, $\left.\mathrm{CDCl}_{3}\right) \delta: 15.0,17.3,64.3,66.8$, 67.9, 77.4, $116.2(\mathrm{~d}, J=22.8 \mathrm{~Hz}), 117.1,126.9$ (d, $J=8.1$ Hz), 133.5 (d, $J=3.4 \mathrm{~Hz}), 160.3$ (d, $J=247.4 \mathrm{~Hz}), 167.6$, 170.7; MS (EI, $70 \mathrm{eV}) \mathrm{m} / z(\%): 324\left(\mathrm{M}^{+}, 22\right), 277$ (18), 234 (50), 205 (16), 187 (100), 159 (24), 110 (5), 95 (11), 73 (22), 45 (59), 32 (27). Anal. calcd for $\mathrm{C}_{15} \mathrm{H}_{17} \mathrm{FN}_{2} \mathrm{O}_{3} \mathrm{~S}$ : C 55.54, H 5.28, N 8.64; found C 55.40, H 5.14, N 8.53.

2-氧基-3-(2,4-二氟)苯胺基-3-甲硫基丙烯酸 (2-乙氧 基乙基)酯(2j): 白色固体，收率 65\%. m.p. 59.0 60.0 ${ }^{\circ} \mathrm{C} ;{ }^{1} \mathrm{H}$ NMR (500 MHz, $\left.\mathrm{CDCl}_{3}\right) \delta: 1.14(\mathrm{t}, J=7.0 \mathrm{~Hz}, 3 \mathrm{H}$, $\left.\mathrm{CH}_{3}\right), 2.34$ (s, $\left.3 \mathrm{H}, \mathrm{SCH}_{3}\right), 3.51$ (q, J=7.0 Hz, $2 \mathrm{H}, \mathrm{OCH}_{2}$ ), $3.65\left(\mathrm{t}, J=5.0 \mathrm{~Hz}, 2 \mathrm{H}, \mathrm{CH}_{2} \mathrm{O}\right), 4.28(\mathrm{t}, J=5.0 \mathrm{~Hz}, 2 \mathrm{H}$, $\left.\mathrm{CO}_{2} \mathrm{CH}_{2}\right), 6.85 \sim 7.30\left(\mathrm{~m}, 3 \mathrm{H}, \mathrm{C}_{6} \mathrm{H}_{3}\right), 11.05(\mathrm{~s}, 1 \mathrm{H}, \mathrm{NH})$; ${ }^{13} \mathrm{C}$ NMR $\left(125 \mathrm{MHz}, \mathrm{CDCl}_{3}\right) \delta: 15.0,17.4,64.4,66.7$, 67.8, 78.6, 104.6 (dd, $J=2.9,23.4 \mathrm{~Hz}), 111.6$ (dd, $J=18.4$, $3.8 \mathrm{~Hz}), 116.7,121.8(\mathrm{dd}, J=8.1,4.1 \mathrm{~Hz}), 128.6$ (d, $J=$ $10.0 \mathrm{~Hz}$ ), 155.7 (dd, $J=239.0,12.1 \mathrm{~Hz}), 160.5$ (dd, $J=$ 239.3, 10.8 Hz), 167.4, 171.7; MS (EI, $70 \mathrm{eV}) \mathrm{m} / \mathrm{z}(\%)$ : 
$342\left(\mathrm{M}^{+}, 20\right), 295$ (16), 252 (71), 205 (86), 177 (19), 152 (9), 113 (7), 73 (26), 45 (58), 32 (100). Anal. calcd for $\mathrm{C}_{15} \mathrm{H}_{16} \mathrm{~F}_{2} \mathrm{~N}_{2} \mathrm{O}_{3} \mathrm{~S}$ : C 52.62, H 4.71, N 8.18; found C 52.58, $\mathrm{H} 4.53, \mathrm{~N} 8.05$.

2-氧基-3-(4-氟-3-氯)苯胺基-3-甲硫基丙烯酸 (2-乙 氧基乙基)酯(2k): 白色固体，收率 67\%. m.p. 99.0 $101.0{ }^{\circ} \mathrm{C} ;{ }^{1} \mathrm{H}$ NMR $\left(500 \mathrm{MHz}, \mathrm{CDCl}_{3}\right) \delta: 1.15$ (t, $J=7.0$ $\left.\mathrm{Hz}, 3 \mathrm{H}, \mathrm{CH}_{3}\right), 2.27$ (s, 3H, $\left.\mathrm{SCH}_{3}\right), 3.52$ (q, $J=7.0 \mathrm{~Hz}, 2 \mathrm{H}$, $\left.\mathrm{OCH}_{2}\right), 3.65\left(\mathrm{t}, J=5.0 \mathrm{~Hz}, 2 \mathrm{H}, \mathrm{CH}_{2} \mathrm{O}\right), 4.28(\mathrm{t}, J=5.0 \mathrm{~Hz}$, $\left.2 \mathrm{H}, \mathrm{CO}_{2} \mathrm{CH}_{2}\right), 7.11 \sim 7.30\left(\mathrm{~m}, 3 \mathrm{H}, \mathrm{C}_{6} \mathrm{H}_{3}\right), 11.30(\mathrm{~s}, 1 \mathrm{H}$, $\mathrm{NH}) ;{ }^{13} \mathrm{C}$ NMR $\left(125 \mathrm{MHz}, \mathrm{CDCl}_{3}\right) \delta: 15.1,17.5,64.6$, $66.9,67.9,78.5,117.1(\mathrm{~d}, J=22.4 \mathrm{~Hz}), 119.4,122.0,125.0$ (d, $J=7.3 \mathrm{~Hz}), 127.4,134.2,156.0(\mathrm{~d}, J=246.3 \mathrm{~Hz})$, 167.5, 170.5; MS (EI, $70 \mathrm{eV}) \mathrm{m} / \mathrm{z}(\%): 358\left(\mathrm{M}^{+}, 27\right), 311$ (20), 268 (71), 239 (15), 221 (100), 193 (14), 168 (9), 129 (17), 73 (39), 45 (84), 29 (33). Anal. calcd for $\mathrm{C}_{15} \mathrm{H}_{16} \mathrm{Cl}$ $\mathrm{FN}_{2} \mathrm{O}_{3} \mathrm{~S}$ : C 50.21, H 4.49, N 7.81; found C 50.17, H 4.32, $\mathrm{N} 7.69$.

\subsection{2 取代苯基腺 $(\mathbf{3 a} \sim 3 \mathbf{k})$ 的合成}

于装有电动搅拌的 $100 \mathrm{~mL}$ 三口反应瓶中, 依次加 入 $8.00 \mathrm{~g}(27 \mathrm{mmol}$ )双 (三氯甲基)碳酸酯(俗称三光气)和 $30 \mathrm{~mL}$ 乙酸乙酯, 待双 (三氯甲基)碳酸酯溶解后, 冰浴 冷却至 $0 \sim 5{ }^{\circ} \mathrm{C}$ 后, 滴加 $54 \mathrm{mmol}$ 取代苯胺溶于 $15 \mathrm{~mL}$ 乙酸乙酯的混合溶液, 滴加完毕后, 室温搅拌 $1 \mathrm{~h}$, 然后 加热回流 3 6 h, 冷却, 浓缩后得到油状液体, 向其中 加入 $20 \mathrm{~mL}$ 丙酮, 冰浴冷却至 $0 \sim 5{ }^{\circ} \mathrm{C}$ 后, 将其滴加到 $11 \mathrm{~mL}(162 \mathrm{mmol})$ 氨水和 $10 \mathrm{~mL}$ 丙酮的混合液中, 室温 摚拌 $12 \mathrm{~h}$, 旋转烝发除去溶剂后加入 $50 \mathrm{~mL}$ 水, 摇匀, 抽滤，烘干滤饼并用乙醇重结晶 ${ }^{[12,13]}$.

\subsubsection{2-氰基-3-取代苯基腿基丙烯酸(2-乙氧基乙基) 酯 $(5 \mathbf{a} \sim 5 \mathbf{k})$ 的合成}

在 $25 \mathrm{~mL}$ 单口烧瓶中依次加入 $0.55 \mathrm{~g}(3.5 \mathrm{mmol})$ 氭 乙酸乙氧乙酯、 $2.9 \mathrm{mmol}$ 的取代苯基脲和 $0.59 \mathrm{~mL}$ ( 3.5 $\mathrm{mmol}$ )原甲酸三乙酯, 于 $105{ }^{\circ} \mathrm{C}$ 反应约 $2 \mathrm{~h}$, 停止反应, 待反应体系冷却析出固体后, 加入少量乙醇, 加热溶 解、冷却、析出固体后抽滤, 固体用母液、石油醚各洗 两次, 烘干滤饼得目标产物 ${ }^{[14]}$. 目标化合物的结构经 ${ }^{1} \mathrm{H}$ NMR, ${ }^{13} \mathrm{C}$ NMR 和质谱分析确证.

2-氧基-3-苯基脲基丙烯酸(2-乙氧基乙基)酯 (5a): 白色片状晶体, 收率 $37 \%$. m.p. $185.3 \sim 185.9{ }^{\circ} \mathrm{C} ;{ }^{1} \mathrm{H}$ NMR $\left(500 \mathrm{MHz}\right.$, DMSO- $\left.d_{6}\right) \delta: 1.13(\mathrm{t}, J=7.0 \mathrm{~Hz}, 3 \mathrm{H}$, $\mathrm{CH}_{3}$ ), 3.50 (q, $J=7.0 \mathrm{~Hz}, 2 \mathrm{H}, \mathrm{OCH}_{2}$ ), 3.65 (t, $J=5.0 \mathrm{~Hz}$, $\left.2 \mathrm{H}, \mathrm{CH}_{2} \mathrm{O}\right), 4.34\left(\mathrm{t}, J=5.0 \mathrm{~Hz}, 2 \mathrm{H}, \mathrm{CO}_{2} \mathrm{CH}_{2}\right), 7.08 \sim 7.52$ (m, $\left.5 \mathrm{H}, \mathrm{C}_{6} \mathrm{H}_{5}\right), 8.23$ (d, $\left.J=12.5 \mathrm{~Hz}, 1 \mathrm{H}, \mathrm{CONH}\right), 10.50$ (s, $1 \mathrm{H}, \mathrm{NHCO}), 10.86(\mathrm{~d}, J=13.0 \mathrm{~Hz}, 1 \mathrm{H},=\mathrm{CH}) ;{ }^{13} \mathrm{C} \mathrm{NMR}$
(125 MHz, DMSO- $\left.d_{6}\right) \delta: 15.1,64.4,65.7,67.5,78.6$, 116.8, 118.8, 123.7, 129.1, 137.9, 148.8, 151.4, 164.8; MS (EI, $70 \mathrm{eV}$ ) $m / z(\%): 303\left(\mathrm{M}^{+}, 12\right), 231$ (12), 165 (8), 139 (12), 119 (100), 112 (38), 93 (70), 72 (81), 45 (30), 39 (15). Anal. calcd for $\mathrm{C}_{15} \mathrm{H}_{17} \mathrm{~N}_{3} \mathrm{O}_{4}$ : C 59.40, H 5.65, N 13.85; found C 59.28, H 5.50, N 13.76 .

2-氧基-3-(2-甲基)苯基腿基丙烯酸(2-乙氧基乙基) 酯(5b): 白色固体, 收率 33\%. m.p. $152.0 \sim 155.0{ }^{\circ} \mathrm{C} ;{ }^{1} \mathrm{H}$ NMR $\left(500 \mathrm{MHz}\right.$, DMSO- $\left.d_{6}\right) \delta: 1.13(\mathrm{t}, J=7.0 \mathrm{~Hz}, 3 \mathrm{H}$, $\left.\mathrm{CH}_{3}\right), 2.26\left(\mathrm{~s}, 3 \mathrm{H}, \mathrm{CH}_{3} \mathrm{C}_{6} \mathrm{H}_{4}\right), 3.50$ (q, $J=7.0 \mathrm{~Hz}, 2 \mathrm{H}$, $\mathrm{OCH}_{2}$ ), 3.65 (t, $J=5.0 \mathrm{~Hz}, 2 \mathrm{H}, \mathrm{CH}_{2} \mathrm{O}$ ), 4.34 (t, $J=5.0 \mathrm{~Hz}$, $\left.2 \mathrm{H}, \mathrm{CO}_{2} \mathrm{CH}_{2}\right), 7.07 \sim 7.67\left(\mathrm{~m}, 4 \mathrm{H}, \mathrm{C}_{6} \mathrm{H}_{4}\right), 8.23$ (d, $J=12.5$ $\mathrm{Hz}, 1 \mathrm{H}, \mathrm{CONH}), 9.71$ (s, 1H, NHCO), 11.09 (d, $J=12.5$ $\mathrm{Hz}, 1 \mathrm{H},=\mathrm{CH}) ;{ }^{13} \mathrm{C}$ NMR $\left(125 \mathrm{MHz}\right.$, DMSO- $\left.d_{6}\right) \delta: 15.0$, $17.9,64.3,65.7,67.4,78.4,116.9,123.0,124.8,126.2$, $129.8,130.4,135.4,149.6,151.4,164.5$; MS (EI, $70 \mathrm{eV}$ ) m/z (\%): $317\left(\mathrm{M}^{+}, 13\right), 245$ (8), 165 (6), 139 (8), 133 (100), 107 (45), 95 (28), 72 (27), 45 (19), 39 (4). Anal. calcd for $\mathrm{C}_{16} \mathrm{H}_{19} \mathrm{~N}_{3} \mathrm{O}_{4}$ : C 60.56, H 6.03, N 13.24; found C 60.44, H 5.94, N 13.17 .

2-氧基-3-(3-甲基)苯基嫝基丙烯酸(2-乙氧基乙基) 酯(5c): 白色固体, 收率 38\%. m.p. 189.0 190.0 ${ }^{\circ} \mathrm{C} ;{ }^{1} \mathrm{H}$ NMR $\left(500 \mathrm{MHz}\right.$, DMSO- $\left.d_{6}\right) \delta: 1.12(\mathrm{t}, J=7.0 \mathrm{~Hz}, 3 \mathrm{H}$, $\mathrm{CH}_{3}$ ), 2.30 (s, $\left.3 \mathrm{H}, \mathrm{CH}_{3} \mathrm{C}_{6} \mathrm{H}_{4}\right), 3.50$ (q, $J=7.0 \mathrm{~Hz}, 2 \mathrm{H}$, $\mathrm{OCH}_{2}$ ), 3.65 (t, $J=5.0 \mathrm{~Hz}, 2 \mathrm{H}, \mathrm{CH}_{2} \mathrm{O}$ ), 4.34 (t, $J=5.0 \mathrm{~Hz}$, $\left.2 \mathrm{H}, \mathrm{CO}_{2} \mathrm{CH}_{2}\right), 6.91 \sim 7.32\left(\mathrm{~m}, 4 \mathrm{H}, \mathrm{C}_{6} \mathrm{H}_{4}\right), 8.22(\mathrm{~d}, J=12.5$ $\mathrm{Hz}, 1 \mathrm{H}, \mathrm{CONH}$ ), 10.43 (s, 1H, NHCO), 10.84 (d, $J=12.5$ $\mathrm{Hz}, 1 \mathrm{H},=\mathrm{CH}) ;{ }^{13} \mathrm{C}$ NMR $\left(125 \mathrm{MHz}\right.$, DMSO- $\left.d_{6}\right) \delta: 15.1$, 21.2, 64.4, 65.7, 67.5, 78.5, 116.0, 116.8, 119.3, 124.4, 124.5, 128.9, 138.4, 148.7, 151.4, 164.7; MS (EI, $70 \mathrm{eV}$ ) m/z (\%): $317\left(\mathrm{M}^{+}, 14\right), 245$ (6), 165 (4), 139 (6), 133 (100), 107 (41), 95 (21), 72 (23), 45 (16), 39 (6). Anal. calcd for $\mathrm{C}_{16} \mathrm{H}_{19} \mathrm{~N}_{3} \mathrm{O}_{4}$ : C 60.56, H 6.03, N 13.24; found $\mathrm{C}$ 60.49 , H 5.92, N 13.13 .

2-氧基-3-(4-甲基)苯基嫝基丙烯酸(2-乙氧基乙基) 酯(5d): 白色片状晶体，收率 33\%. m.p. 183.0 185.0 ${ }^{\circ} \mathrm{C}$; ${ }^{1} \mathrm{H}$ NMR (500 MHz, DMSO- $d_{6}$ ) $\delta: 1.12$ (t, $J=7.0 \mathrm{~Hz}$, $\left.3 \mathrm{H}, \mathrm{CH}_{3}\right), 2.26$ (s, $\left.3 \mathrm{H}, \mathrm{CH}_{3} \mathrm{C}_{6} \mathrm{H}_{4}\right), 3.50$ (q, $J=7.0 \mathrm{~Hz}, 2 \mathrm{H}$, $\mathrm{OCH}_{2}$ ), 3.64 (t, $J=4.5 \mathrm{~Hz}, 2 \mathrm{H}, \mathrm{CH}_{2} \mathrm{O}$ ), 4.34 (t, $J=4.5 \mathrm{~Hz}$, $\left.2 \mathrm{H}, \mathrm{CO}_{2} \mathrm{CH}_{2}\right), 7.15 \sim 7.39\left(\mathrm{~m}, 4 \mathrm{H}, \mathrm{C}_{6} \mathrm{H}_{4}\right), 8.21(\mathrm{~d}, J=12.5$ $\mathrm{Hz}, 1 \mathrm{H}, \mathrm{CONH}), 10.40$ (s, 1H, NHCO), 10.82 (d, $J=12.5$ $\mathrm{Hz}, 1 \mathrm{H},=\mathrm{CH}) ;{ }^{13} \mathrm{C}$ NMR $\left(125 \mathrm{MHz}\right.$, DMSO- $\left.d_{6}\right) \delta: 15.5$, 20.9, 64.8, 66.1, 67.9, 78.8, 117.3, 119.3, 129.9, 133.2, 135.8, 149.2, 151.8, 165.2; MS (EI, $70 \mathrm{eV}) \mathrm{m} / \mathrm{z}(\%): 317$ ( $\left.\mathrm{M}^{+}, 13\right), 245$ (2), 165 (5), 139 (5), 133 (100), 107 (47), 95 
(17), 72 (12), 45 (14), 39 (4). Anal. calcd for $\mathrm{C}_{16} \mathrm{H}_{19} \mathrm{~N}_{3} \mathrm{O}_{4}$ : C 60.56, H 6.03, N 13.24; found C 60.39, H 5.87, N 13.12.

2-氧基-3-(2-氯)苯基腿基丙烯酸(2-乙氧基乙基)酯 (5e): 白色固体, 收率 28\%. m.p. 169.0 170.3 ${ }^{\circ} \mathrm{C} ;{ }^{1} \mathrm{H}$ NMR $\left(500 \mathrm{MHz}\right.$, Acetone- $\left.d_{6}\right) \delta: 1.15(\mathrm{t}, J=7.0 \mathrm{~Hz}, 3 \mathrm{H}$, $\mathrm{CH}_{3}$ ), 3.53 (q, $J=7.0 \mathrm{~Hz}, \mathrm{OCH}_{2}$ ), 3.70 (t, $J=5.0 \mathrm{~Hz}, 2 \mathrm{H}$, $\left.\mathrm{CH}_{2} \mathrm{O}\right), 4.37\left(\mathrm{t}, J=5.0 \mathrm{~Hz}, 2 \mathrm{H}, \mathrm{CO}_{2} \mathrm{CH}_{2}\right), 7.18 \sim 8.14(\mathrm{~m}$, $\left.4 \mathrm{H}, \mathrm{C}_{6} \mathrm{H}_{4}\right), 8.29(\mathrm{~s}, 1 \mathrm{H}, \mathrm{CONH}), 9.47(\mathrm{~s}, 1 \mathrm{H}, \mathrm{NHCO})$, $10.83(\mathrm{~s}, 1 \mathrm{H},=\mathrm{CH}) ;{ }^{13} \mathrm{C}$ NMR $\left(125 \mathrm{MHz}\right.$, Acetone- $\left.d_{6}\right) \delta$ : 15.5, 65.5, 67.0, 68.6, 81.5, 116.8, 124.6, 125.5, 126.5, 128.5, 130.4, 135.5, 150.4, 151.8, 165.9; MS (EI, $70 \mathrm{eV}$ ) $\mathrm{m} / \mathrm{z}$ (\%): $337\left(\mathrm{M}^{+}, 5\right), 302$ (12), 230 (8), 212 (8), 153 (93), 127 (70), 112 (41), 95 (100), 90 (47), 72 (92), 45 (32). Anal. calcd for $\mathrm{C}_{15} \mathrm{H}_{16} \mathrm{ClN}_{3} \mathrm{O}_{4}$ : C 53.34, H 4.77, $\mathrm{N}$ 12.44; found $\mathrm{C} 53.25, \mathrm{H} 4.62, \mathrm{~N} 12.33$.

2-氭基-3-(3-氯)苯基脲基丙烯酸(2-乙氧基乙基)酯 (5f): 淡黄色固体, 收率 30\%. m.p. 189.2 190.3 ${ }^{\circ} \mathrm{C} ;{ }^{1} \mathrm{H}$ NMR $\left(500 \mathrm{MHz}\right.$, DMSO- $\left.d_{6}\right) \delta: 1.12(\mathrm{t}, J=7.0 \mathrm{~Hz}, 3 \mathrm{H}$, $\mathrm{CH}_{3}$ ), 3.50 (q, $J=7.0 \mathrm{~Hz}, 2 \mathrm{H}, \mathrm{OCH}_{2}$ ), 3.65 (t, $J=5.0 \mathrm{~Hz}$, $\left.2 \mathrm{H}, \mathrm{CH}_{2} \mathrm{O}\right), 4.35\left(\mathrm{t}, J=5.0 \mathrm{~Hz}, 2 \mathrm{H}, \mathrm{CO}_{2} \mathrm{CH}_{2}\right), 7.15 \sim 7.70$ (m, 4H, $\left.\mathrm{C}_{6} \mathrm{H}_{4}\right), 8.22$ (d, J=12.5 Hz, 1H, CONH), 10.66 (s, $1 \mathrm{H}, \mathrm{NHCO}), 10.84(\mathrm{~d}, J=12.5 \mathrm{~Hz}, 1 \mathrm{H},=\mathrm{CH}) ;{ }^{13} \mathrm{C} \mathrm{NMR}$ (125 MHz, DMSO- $d_{6}$ ) $\delta$ : 15.0, 64.4, 65.6, 67.3, 79.1, $116.5,117.3,118.3,123.3,130.7,133.3,139.3,148.8$, 151.1, 164.6; MS (EI, $70 \mathrm{eV}) \mathrm{m} / \mathrm{z}(\%): 337\left(\mathrm{M}^{+}, 6\right), 165$ (13), 153 (71), 127 (79), 112 (45), 95 (65), 90 (20), 72 (100), 45 (42). Anal. calcd for $\mathrm{C}_{15} \mathrm{H}_{16} \mathrm{ClN}_{3} \mathrm{O}_{4}$ : C 53.34, $\mathrm{H}$ 4.77, N 12.44; found C 53.28, H 4.66, N 12.39 .

2-氧基-3-(4-氯)苯基腿基丙烯酸(2-乙氧基乙基)酯 (5g): 白色晶体, 收率 $25 \%$. m.p. $192.4 \sim 193.4{ }^{\circ} \mathrm{C} ;{ }^{1} \mathrm{H}$ NMR $\left(500 \mathrm{MHz}\right.$, Acetone- $\left.d_{6}\right) \delta: 1.15(\mathrm{t}, J=7.0 \mathrm{~Hz}, 3 \mathrm{H}$, $\mathrm{CH}_{3}$ ), 3.53 (q, $J=7.0 \mathrm{~Hz}, 2 \mathrm{H}, \mathrm{OCH}_{2}$ ), 3.70 (t, $J=5.0 \mathrm{~Hz}$, $\left.2 \mathrm{H}, \mathrm{CH}_{2} \mathrm{O}\right), 4.38\left(\mathrm{t}, J=5.0 \mathrm{~Hz}, 2 \mathrm{H}, \mathrm{CO}_{2} \mathrm{CH}_{2}\right), 7.38 \sim 7.62$ (m, $\left.4 \mathrm{H}, \mathrm{C}_{6} \mathrm{H}_{4}\right), 8.27$ (s, $\left.1 \mathrm{H}, \mathrm{CONH}\right), 9.81(\mathrm{~s}, 1 \mathrm{H}, \mathrm{NHCO})$, $10.66(\mathrm{~s}, 1 \mathrm{H},=\mathrm{CH}) ;{ }^{13} \mathrm{C}$ NMR $\left(125 \mathrm{MHz}\right.$, Acetone- $\left.d_{6}\right) \delta$ : $15.5,65.6,67.0,68.6,81.1,116.7,121.5,129.2,129.9$, 138.0, 149.7, 152.0, 166.5; MS (EI, $70 \mathrm{eV}) \mathrm{m} / \mathrm{z}: 337\left(\mathrm{M}^{+}\right.$, 6), 165 (13), 153 (70), 127 (75), 112 (46), 95 (63), 90 (21), 72 (100), 45 (42). Anal. calcd for $\mathrm{C}_{15} \mathrm{H}_{16} \mathrm{ClN}_{3} \mathrm{O}_{4}$ : C 53.34, H 4.77, N 12.44; found C 53.22, H 4.72, N 12.36 .

2-氧基-3-(2-氟)苯基脲基丙烯酸(2-乙氧基乙基)酯 (5h): 白色固体, 收率 31\%. m.p. 164.0 165.7 ${ }^{\circ} \mathrm{C} ;{ }^{1} \mathrm{H}$ NMR $\left(500 \mathrm{MHz}\right.$, Acetone- $\left.d_{6}\right) \delta: 1.15(\mathrm{t}, J=7.0 \mathrm{~Hz}, 3 \mathrm{H}$, $\mathrm{CH}_{3}$ ), 3.53 (q, $\left.J=7.0 \mathrm{~Hz}, 2 \mathrm{H}, \mathrm{OCH}_{2}\right), 3.70$ (t, $J=5.0 \mathrm{~Hz}$, $\left.2 \mathrm{H}, \mathrm{CH}_{2} \mathrm{O}\right), 4.37\left(\mathrm{t}, J=5.0 \mathrm{~Hz}, 2 \mathrm{H}, \mathrm{CO}_{2} \mathrm{CH}_{2}\right), 7.15 \sim 8.24$ (m, 4H, $\left.\mathrm{C}_{6} \mathrm{H}_{4}\right), 8.29$ (d, $\left.J=10.5 \mathrm{~Hz}, 1 \mathrm{H}, \mathrm{CONH}\right), 9.72$ (s, $1 \mathrm{H}, \mathrm{NHCO}), 10.82(\mathrm{~d}, J=9.0 \mathrm{~Hz}, 1 \mathrm{H},=\mathrm{CH}) ;{ }^{13} \mathrm{C} \mathrm{NMR}$ (125 MHz, Acetone- $d_{6}$ ) $\delta: 15.5,65.5,67.0,68.7,81.4$, 116.0 (d, $J=18.9 \mathrm{~Hz}), 116.8,122.9,125.5(\mathrm{~d}, J=3.5 \mathrm{~Hz})$, 125.7 (d, $J=7.5 \mathrm{~Hz}), 126.9$ (d, $J=10.5 \mathrm{~Hz}), 150.1,151.8$, 153.0 (d, $J=242.0 \mathrm{~Hz}), 166.0$; MS (EI, $70 \mathrm{eV}) \mathrm{m} / \mathrm{z}(\%)$ : $321\left(\mathrm{M}^{+}, 6\right), 249$ (9), 207 (14), 137 (79), 121 (5), 111 (100), 95 (96), 72 (81), 59 (52), 45 (32). Anal. calcd for $\mathrm{C}_{15} \mathrm{H}_{16} \mathrm{FN}_{3} \mathrm{O}_{4}$ : C 56.07, H 5.02, N 13.08; found C 56.02, $\mathrm{H}$ 4.95, N 12.94.

2-氰基-3-(4-氟)苯基腿基丙烯酸(2-乙氧基乙基)酯 (5i): 白色固体, 收率 32\%. m.p. 194.5 195.6 ${ }^{\circ} \mathrm{C} ;{ }^{1} \mathrm{H}$ NMR $\left(500 \mathrm{MHz}\right.$, Acetone- $\left.d_{6}\right) \delta: 1.15(\mathrm{t}, J=7.0 \mathrm{~Hz}, 3 \mathrm{H}$, $\left.\mathrm{CH}_{3}\right), 3.53$ (q, $\left.J=7.0 \mathrm{~Hz}, 2 \mathrm{H}, \mathrm{OCH}_{2}\right), 3.70(\mathrm{t}, J=5.0 \mathrm{~Hz}$, $\left.2 \mathrm{H}, \mathrm{CH}_{2} \mathrm{O}\right), 4.38\left(\mathrm{t}, J=5.0 \mathrm{~Hz}, 2 \mathrm{H}, \mathrm{CO}_{2} \mathrm{CH}_{2}\right), 7.12 \sim 7.63$ (m, 4H, $\left.\mathrm{C}_{6} \mathrm{H}_{4}\right), 8.28(\mathrm{~d}, J=6.5 \mathrm{~Hz}, 1 \mathrm{H}, \mathrm{CONH}), 9.73$ (s, $1 \mathrm{H}, \mathrm{NHCO}), 10.65(\mathrm{~s}, 1 \mathrm{H},=\mathrm{CH}) ;{ }^{13} \mathrm{C}$ NMR $(125 \mathrm{MHz}$, Acetone- $\left.d_{6}\right) \delta: 15.5,65.6,67.0,68.7,80.8,116.3,116.5$, $121.9,135.3,149.8,152.1,159.1$ (d, $J=239.6 \mathrm{~Hz}), 166.5$; MS (EI, $70 \mathrm{eV}) \mathrm{m} / z$ (\%): $321\left(\mathrm{M}^{+}, 13\right), 249$ (9), 165 (13), 137 (87), 121 (6), 111 (100), 95 (92), 72 (58), 59 (40), 45 (42). Anal. calcd for $\mathrm{C}_{15} \mathrm{H}_{16} \mathrm{FN}_{3} \mathrm{O}_{4}$ : C 56.07, H 5.02, N 13.08; found C 55.92, H 4.93, N 12.90 .

2-氧基-3-(2,4-二氟)苯基脲基丙烯酸(2-乙氧基乙基) 酯(5j): 白色固体, 收率 26\%. m.p. 191.0 192.0 ${ }^{\circ} \mathrm{C} ;{ }^{1} \mathrm{H}$ NMR $\left(500 \mathrm{MHz}\right.$, Acetone- $\left.d_{6}\right) \delta: 1.15(\mathrm{t}, J=7.0 \mathrm{~Hz}, 3 \mathrm{H}$, $\left.\mathrm{CH}_{3}\right), 3.53$ (q, $J=7.0 \mathrm{~Hz}, 2 \mathrm{H}, \mathrm{OCH}_{2}$ ), 3.70 (t, $J=5.0 \mathrm{~Hz}$, $\left.2 \mathrm{H}, \mathrm{CH}_{2} \mathrm{O}\right), 4.37\left(\mathrm{t}, J=5.0 \mathrm{~Hz}, 2 \mathrm{H}, \mathrm{CO}_{2} \mathrm{CH}_{2}\right), 7.04 \sim 8.21$ (m, $\left.3 \mathrm{H}, \mathrm{C}_{6} \mathrm{H}_{3}\right), 8.28$ (d, J=9.0 Hz, 1H, CONH), 9.69 (s, $1 \mathrm{H}, \mathrm{NHCO}), 10.79$ (d, $J=9.0 \mathrm{~Hz}, 1 \mathrm{H},=\mathrm{CH}) ;{ }^{13} \mathrm{C} \mathrm{NMR}$ $\left(125 \mathrm{MHz}\right.$, Acetone- $\left.d_{6}\right) \delta$ : 15.5, 65.5, 67.0, 68.7, 81.4, $104.4(\mathrm{dd}, J=3.5,23.4 \mathrm{~Hz}), 112.0(\mathrm{dd}, J=18.3,3.5 \mathrm{~Hz})$, $116.8,123.6,124.5,150.3,151.8,166.1$; MS (EI, $70 \mathrm{eV}$ ) $\mathrm{m} / \mathrm{z}(\%): 339\left(\mathrm{M}^{+}, 5\right), 267$ (6), 211 (9), 165 (11), 155 (46), 129 (100), 112 (13), 95 (63), 72 (58), 59 (40), 45 (30). Anal. calcd for $\mathrm{C}_{15} \mathrm{H}_{15} \mathrm{~F}_{2} \mathrm{~N}_{3} \mathrm{O}_{4}$ : C 53.10, H 4.46, N 12.38; found $\mathrm{C} 53.00, \mathrm{H} 4.31, \mathrm{~N} 12.24$.

2-氧基-3-(4-氟-3-氯)苯基腿基丙烯酸 (2-乙氧基乙 基)酯(5k): 白色固体，收率 24\%. m.p. 189.0 191.0 ${ }^{\circ} \mathrm{C}$; ${ }^{1} \mathrm{H}$ NMR (500 MHz, DMSO- $\left.d_{6}\right) \delta: 1.13(\mathrm{t}, J=7.0 \mathrm{~Hz}, 3 \mathrm{H}$, $\mathrm{CH}_{3}$ ), 3.50 (q, $J=7.0 \mathrm{~Hz}, 2 \mathrm{H}, \mathrm{OCH}_{2}$ ), 3.65 (t, $J=4.5 \mathrm{~Hz}$, $2 \mathrm{H}, \mathrm{CH}_{2} \mathrm{O}$ ), 4.35 (t, $\left.J=5.0 \mathrm{~Hz}, 2 \mathrm{H}, \mathrm{CO}_{2} \mathrm{CH}_{2}\right), 7.33 \sim 7.79$ (m, 3H, $\left.\mathrm{C}_{6} \mathrm{H}_{3}\right), 8.15$ (d, $\left.J=12.5 \mathrm{~Hz}, 1 \mathrm{H}, \mathrm{CONH}\right), 10.65$ (s, $1 \mathrm{H}, \mathrm{NHCO}), 10.81(\mathrm{~d}, J=12.5 \mathrm{~Hz}, 1 \mathrm{H},=\mathrm{CH}) ;{ }^{13} \mathrm{C} \mathrm{NMR}$ $\left(125 \mathrm{MHz}, \mathrm{DMSO}-d_{6}\right) \delta: 14.9,64.4,65.6,67.3,79.1$, 
116.5, 117.1 (d, $J=21.9 \mathrm{~Hz}), 119.2$ (d, $J=6.5 \mathrm{~Hz}), 119.4$

$(\mathrm{d}, J=18.4 \mathrm{~Hz}), 120.2,135.1(\mathrm{~d}, J=3.5 \mathrm{~Hz}), 148.9,151.1$, 152.4 (d, $J=241.6 \mathrm{~Hz}$ ), 164.6; MS (EI, $70 \mathrm{eV}) \mathrm{m} / \mathrm{z}(\%)$ : 355 ( $\left.\mathrm{M}^{+}, 13\right), 283$ (10), 238 (38), 207 (80), 171 (100), 145 (41), 108 (76), 95 (92), 72 (66), 59 (49), 45 (58). Anal. calcd for $\mathrm{C}_{15} \mathrm{H}_{15} \mathrm{ClFN}_{3} \mathrm{O}_{4}$ : C 50.64, $\mathrm{H} 4.25, \mathrm{~N} 11.81$; found C 50.35, H 4.17, N 11.73 .

\section{References}

[1] Jin, H.-L.; Zhong, H.-M.; Song, B.-A. Chin. J. Synth. Chem. 2005, 13, 113 (in Chinese). (金红林，钟惠民，宋宝安，合成化学, 2005, 13, 113.)

[2] Zhang, H.-P.; Song, B.-A.; Wang, X.-Y. Chin. J. Org. Chem. 2008, 28, 17 (in Chinese). (张慧苹, 宋宝安, 王献友, 有机化学, 2008, 28, 17.)

[3] Wang, L.-G.; Wang, F.-Y.; Diao, Y.-M. Chin. J. Org. Chem. 2005, 25, 1254 (in Chinese) (王龙根, 王风云, 刀亚梅, 有机化学, 2005, 25, 1254.)

[4] McFadden, H. G.; Phillips, J. N. J. Z. Naturforsch. 1990, 45C, 196.

[5] Banham, W. K.; Huppatz, J. N.; Phillips, J. N. J. Z. Naturforsch. 1993, $48 C, 136$

[6] Liu, Y.-X.; Li, H.; Zhao, Q.-Q. Chin. J. Org. Chem. 2006, 26, 1232 (in Chinese)

(刘玉秀, 李姮, 赵奇奇, 有机化学, 2006, 26, 1232.)

[7] Zhou, X.-M.; Yu, L.-M.; Gao, Y. Chin. J. Org. Chem. 2006, 26, 337 (in Chinese).

(邹小毛, 郁丽敏, 高颖, 有机化学, 2006, 26, 337.)

[8] Wang, T.-T.; Bing, G.-F.; Zhang, X. Chem. J. Chin. Univ. 2010, 31, 708 (in Chinese) (王婷婷，邴贵芳，张欣，高等学校化学学报，2010, 31, 708.)

[9] Liu, H.-Y.; Lu, R.-J.; Yang, H.-Z. Chemisty 1997, (6), 14 (in Chinese). (刘华银，陆荣健，杨华铮，化学通报, 1997, (6), 14.)

[10] Liu, H.-Y.; Sha, Y.-L.; Lu, R.-J. Chin. Sci. Bull. 1998, 43, 397 (in Chinese). (刘华银, 沙印林, 陆荣健, 科学通报, 1998, 43, 397.)

[11] Wang, Q. M.; Li H., Li, Y. H. J. Agric. Food Chem. 2004, 52, 1918.

[12] Xue, Y.; Wu, S.-Z.; Peng, A.-D. Chin. J. Org. Chem. 2002, 22, 529 (in Chinese). (薛燕，吴思忠，彭爱东，有机化学，2002，22，529.)

[13] Wang, X.-H.; He, Y.-N.; Pan, M.-D. Appl. Chem. Ind. 2008, 37, 1019 (in Chinese).

(王向辉，贺永宁，盘茂东，应用化工， 2008，37，1019.)

[14] Senda, S.; Hirota, K.; Notani, J. Chem. Pharm. Bull. 1972, 20, 1380. 